hep-th/0209002

MIT-CTP-3300

HUTP-02/A042

\title{
Operator Mixing and the BMN Correspondence
}

\author{
Neil R. Constable, ${ }^{1}$ Daniel Z. Freedman, ${ }^{2}$ Matthew Headrick, ${ }^{3}$ and Shiraz Minwalla ${ }^{3}$ \\ 2 Department of Mathematics, \\ 1,2 Center for Theoretical Physics and Laboratory for Nuclear Science \\ Massachusetts Institute of Technology \\ Cambridge, Ma. 02139 \\ constabl@lns.mit.edu, dzf@math.mit.edu \\ 3 Jefferson Physical Laboratory \\ Department of Physics \\ Harvard University \\ Cambridge, MA 02138 \\ headrick@pascal.harvard.edu, minwalla@fas.harvard.edu
}

\begin{abstract}
In this note we update the discussion of the BMN correspondence and string interactions in hep-th/0205089 to incorporate the effects of operator mixing. We diagonalize the matrix of two point functions of single and double trace operators, and compute the eigen-operators and their anomalous dimensions to order $g_{2}^{2} \lambda^{\prime}$. Operators in different $\mathrm{R}$ symmetry multiplets remain degenerate at this order; we propose this is a consequence of supersymmetry. We also calculate the corresponding energy shifts in string theory, and find a discrepancy with field theory results, indicating possible new effects in light-cone string field theory.
\end{abstract}




\section{Contents}

1. Introduction . . . . . . . . . . . . . . . . . . . . . . . . . . . . . . . . . 1

2. Anomalous dimensions of BMN eigen-operators . . . . . . . . . . . . . . . . . 3

2.1. Two-point functions, three-point functions, and mixing . . . . . . . . . . . . . 3

2.2. Construction of eigen-operators . . . . . . . . . . . . . . . . . . . . . . . 6

2.3. Consistency conditions from three-point functions . . . . . . . . . . . . . . . 6

2.4. Anomalous dimensions of BMN eigen-operators . . . . . . . . . . . . . . . . 7

3. String field theory revisited . . . . . . . . . . . . . . . . . . . . . . . . . . . 9

Appendix A. The space-time structure of two- and three-point functions . . . . . . . . 11

Appendix B. Absence of mixing with BPS double-trace operators . . . . . . . . . . . . 12

Appendix C. Diagonalization procedure . . . . . . . . . . . . . . . . . . . . . . . 12

Appendix D. Deconstruction identities . . . . . . . . . . . . . . . . . . . . . . . 14

Appendix E. Degenerate operators and mixing . . . . . . . . . . . . . . . . . . . . 16

\section{Introduction}

Several aspects of the correspondence [1] between operators of $\mathcal{N}=4 S U(N)$ superYang-Mills theory at large R-charge $J$ and type IIB string theory in a pp-wave background geometry [2, 3] have been investigated in the recent literature. The map between field theory operators and free string theory in the pp-wave background was established by [1]. In our paper [1] we attempted to extend this map to a correspondence between Yang-Mills correlators and interactions in the pp-wave background. In this note we will update and correct the discussion presented in [4].

Field theory in the BMN limit, $N \rightarrow \infty, J \rightarrow \infty$ with $J^{2} / N$ fixed, appears to be governed by two parameters [1, [4, [6, 6]: an effective gauge coupling $\lambda^{\prime}=g_{\mathrm{YM}}^{2} N / J^{2}$, and an effective genus counting parameter $g_{2}=J^{2} / N$. These quantities can be expressed in terms of the string scale $\alpha^{\prime}$ and coupling $g_{\mathrm{s}}$, light-cone momentum $p^{+}$, and transverse string mass $\mu$ as

$$
\lambda^{\prime}=\frac{1}{\left(\mu p^{+} \alpha^{\prime}\right)^{2}}, \quad g_{2}=4 \pi g_{\mathrm{s}}\left(\mu p^{+} \alpha^{\prime}\right)^{2} .
$$

In [4] we proposed that free three-point functions of the BMN operators

$$
O_{n}^{J} \equiv \frac{1}{\sqrt{J N^{J+2}}} \sum_{l=0}^{J} e^{2 \pi i n l / J} \operatorname{Tr}\left(\phi Z^{l} \psi Z^{J-l}\right)
$$

are related to matrix elements of the string field theory light-cone Hamiltonian according to

$$
\left\langlei \left| H_{\text {int }}(|j\rangle \otimes|k\rangle)=\mu g_{2}\left(\Delta_{i}-\Delta_{j}-\Delta_{k}\right) C_{i j k},\right.\right.
$$


where $|i\rangle,|j\rangle,|k\rangle$ are the dual string states and $\left\langle O_{i} \bar{O}_{j} \bar{O}_{k}\right\rangle=g_{2} C_{i j k}$ (we have factored out the trivial space time dependence). This proposal was intended to describe string transitions in which the initial and final states have the same number of excitations, so that $\Delta_{i}-\Delta_{j}-\Delta_{k}$ is of order $\lambda^{\prime}$. The proposal applies in the limit of large $\mu$, corresponding to weak coupling in field theory. The RHS of (1.3) was computed in 4 by a direct evaluation of the free three-point coupling $C_{i j k}$. Subsequently, the LHS of (1.3) was obtained in [7] (see also [8,9, 10]) as the large $\mu$ limit of the string field theory interaction $H_{\text {int }}$ in the pp-wave background [11]. Results of [7] confirm the proposal (1.3), which thus appears to be on firm ground.

We claimed in [4] that the proposal (1.3) obeys a nontrivial consistency check, which we now review. Utilizing Hamiltonian matrix elements from (1.3), and second-order nondegenerate perturbation theory, we computed the one loop mass renormalization of excited pp-wave string states. We then compared the result to an explicit computation of the order $\lambda^{\prime} g_{2}^{2}$ correction to the anomalous dimension of the corresponding BMN operator. In [4] we reported agreement between these two computations.

In this note we will point out that this agreement is in fact spurious. Firstly our result for the anomalous dimension computed in our paper had the wrong sign (this was pointed out to us by the authors of [5]). Secondly our computation was incomplete, in that it did not take into account mixing between single- and double-trace BMN operators. As we will describe in this paper, this mixing alters the result for the anomalous dimension at the order under consideration. In this note we will present a corrected result for the anomalous dimension of BMN operators at order $\lambda^{\prime} g_{2}^{2}$.2

Surprisingly, this corrected anomalous scaling dimension does not match the result of the analogous perturbative calculation on the string theory side, as we show in section 3. The discrepancy could be due to presence of a quadratic contact term of order $g_{2}^{2}$ in the string field theory Hamiltonian. This is an important issue.

1 In Appendix D we show that the relation referred to in [幽 as the "unitarity check" (with the sign corrected) in fact follows purely from field theory considerations, without making use of the BMN duality.

2 Operator mixing was first suggested in the BMN context in 12]. A preliminary version of the present work was presented by one of us (S.M.) at the conference Strings 2002. As we were drafting this note, the paper [13] was submitted to the archive with similar methodology and results, for a larger class of operators. One of us (D.Z.F.) acknowledges useful discussions with G. Semenoff at the Aspen Center for Physics. Mixing is also discussed in the very recent paper [14]. 


\section{Anomalous dimensions of BMN eigen-operators}

The BMN limit preserves an $S O(4)$ R-symmetry group. The complex impurity fields $\phi$ and $\psi$ transform in the fundamental representation of an $S U(2)$ subgroup, and operators containing two such impurities transform in the $\mathbf{2} \otimes \mathbf{2}=\mathbf{1} \oplus \mathbf{3}$ representation. Our calculations thus split into independent sectors for the two representations. 3 For example, if we form the linear combinations

$$
O_{n}^{ \pm J}=\frac{1}{\sqrt{2}}\left(O_{n}^{J} \pm O_{-n}^{J}\right)
$$

of the operators (1.2), then $O_{n}^{+J}$ is a member of a triplet, while $O_{n}^{-J}$ is a singlet. In this section we will determine, to order $\lambda^{\prime} g_{2}^{2}$, the anomalous dimension of the eigen-operators $\tilde{O}_{n}^{ \pm J}$ that reduce to $O_{n}^{ \pm J}$ at $g_{2}=0$. A priori the results could have been different for the two representations. We find instead that the degeneracy persists to this order in $g_{2}$; at the end of this subsection we will argue that this degeneracy is a consequence of supersymmetry.

The mixing problem requires the diagonalization of the matrix of two-point functions $\left\langle O_{(1)} \bar{O}_{(1)}\right\rangle,\left\langle O_{(1)} \bar{O}_{(2)}\right\rangle,\left\langle O_{(2)} \bar{O}_{(2)}\right\rangle$ of single- and double-trace operators including free and order $\lambda^{\prime}$ terms. The mixed two-point functions are of order $g_{2}$. To obtain the eigenoperators to this order we need only the order $g_{2}^{0}$ parts of $\left\langle O_{(1)} O_{(1)}\right\rangle$ and $\left\langle O_{(2)} O_{(2)}\right\rangle$. The eigen-operators are obtained in subsection 2.1, in a treatment which emphasizes the relation of two- and three-point functions and the issue of correct conformal behavior. In subsection 2.2 we go on to find the order $\lambda^{\prime} g_{2}^{2}$ correction to the anomalous dimension of the eigenoperators $\tilde{O}_{n}^{ \pm J}$ which requires the known [5, [4] order $g_{2}^{2}$ parts of $\left\langle O_{(1)} O_{(1)}\right\rangle$. As discussed in Appendix $\mathrm{E}$, in these calculations we make the assumption that the single-trace operators do not mix with degenerate triple-trace operators, which is not yet justified by calculation of the relevant two-point functions. However, the mode $n=1$ is not degenerate with any multi-trace operators, so results for $n=1$ are safe from this danger.

\subsection{Two-point functions, three-point functions, and mixing}

The two-point functions between single-trace BMN operators $O_{n}^{J}$ was computed to lowest order in $g_{2}$ in [司:

$$
\left\langle O_{n}^{ \pm J} \bar{O}_{m}^{ \pm J}\right\rangle=\delta_{n m}\left(1-\lambda^{\prime} m^{2} \ln \left(x^{2} \Lambda^{2}\right)\right), \quad\left\langle O_{n}^{+J} \bar{O}_{m}^{-J}\right\rangle=0 .
$$

3 We thank H. Verlinde and L. Motl for pointing this out to us. 
In order to establish some notation that will be useful below we may express two-point functions between BMN operators of charge $J_{1}<J$ as

$$
\left\langle O_{n}^{ \pm J_{1}} \bar{O}_{m}^{ \pm J_{1}}\right\rangle=\delta_{n m}\left(1-\lambda^{\prime} k^{2} \ln \left(x^{2} \Lambda^{2}\right)\right)
$$

where $k=J m / J_{1}=m / s$, and we have defined $s=J_{1} / J$ (this was denoted $y$ in [4]).

We now turn to the construction of the matrix of two-point functions between singletrace and double-trace BMN operators. We will find it convenient to first compute, to order $\lambda^{\prime} g_{2}$, the three-point functions of three single-trace BMN operators located at distinct spatial points; the requisite two-point functions may then be obtained allowing two of the insertions in the three-point function to approach each other.

The Feynman graphs that contribute to the three-point function between $O_{n}^{J}, O_{m}^{J_{1}}$, and $O^{J-J_{1}} \equiv \operatorname{Tr}\left(Z^{J-J_{1}}\right) / \sqrt{\left(J-J_{1}\right) N^{J-J_{1}}}$ at order $\lambda^{\prime}$ are of two kinds, with different space time dependence. This gives the following general structure for the three-point function:

$$
\left\langle O_{n}^{J}\left(x_{1}\right) \bar{O}_{m}^{J_{1}}\left(x_{2}\right) \bar{O}^{J-J_{1}}\left(x_{3}\right)\right\rangle=g_{2} C_{n m s}\left[1-\lambda^{\prime}\left(a_{n k} \ln \left(x_{12} \Lambda\right)^{2}+b_{n k} \ln \left(\frac{x_{13} x_{12} \Lambda}{x_{23}}\right)\right)\right] .
$$

Here $C_{n m s}$ is the free three-point function between the relevant operators which was computed in [4],

$$
C_{n m s}=\sqrt{\frac{1-s}{J s}} \frac{\sin ^{2}(\pi n s)}{\pi^{2}(n-k)^{2}} .
$$

Evaluation of the Feynman diagrams gives the values

$$
a_{n k}=k^{2}, \quad b_{n k}=n(n-k) .
$$

but we find it useful to regard $a_{n k}$ and $b_{n k}$ as unspecified parameters in much of this section.

Note that (2.4), with the values of $a_{n k}$ and $b_{n k}$ given in (2.6) does not take the form dictated by conformal invariance for the three-point functions of three operators of anomalous dimension $n^{2} \lambda^{\prime}, k^{2} \lambda^{\prime}$, and 0 respectively. This already indicates that $O_{n}^{J}$ does not have well defined scaling dimension; we will see this in more detail below.

Transforming (2.4) to the $O_{n}^{ \pm J}$ basis, and using the properties $a_{n k}=a_{-n,-k}, b_{n k}=$ $b_{-n,-k}$, we can rewrite the above three-point function as

$$
\left\langle O_{n}^{ \pm J}\left(x_{1}\right) \bar{O}_{m}^{ \pm J_{1}}\left(x_{2}\right) \bar{O}^{J-J_{1}}\left(x_{3}\right)\right\rangle=g_{2} C_{n m s}^{ \pm}\left[1-\lambda^{\prime}\left(a_{n k}^{ \pm} \ln \left(x_{12} \Lambda\right)^{2}+b_{n k}^{ \pm} \ln \left(\frac{x_{13} x_{12} \Lambda}{x_{23}}\right)\right)\right]
$$


and

$$
\left\langle O_{n}^{ \pm J}\left(x_{1}\right) \bar{O}_{m}^{\mp J_{1}}\left(x_{2}\right) \bar{O}^{J-J_{1}}\left(x_{3}\right)\right\rangle=0
$$

where

$$
\begin{aligned}
& C_{n m s}^{+}=C_{n m s}+C_{-n, m, s}=H_{n k s} \frac{2\left(n^{2}+k^{2}\right)}{\left(n^{2}-k^{2}\right)^{2}}, \\
& C_{n m s}^{-}=C_{n m s}-C_{-n, m, s}=H_{n k s} \frac{4 n k}{\left(n^{2}-k^{2}\right)^{2}}, \\
& H_{n k s}=\sqrt{\frac{1-s}{J s}} \frac{\sin ^{2}(n \pi s)}{\pi^{2}} .
\end{aligned}
$$

When the values in (2.6) are used

$$
\begin{aligned}
& a_{n k}^{ \pm}=k^{2}, \\
& b_{n k}^{+}=\frac{n^{2}\left(n^{2}-k^{2}\right)}{n^{2}+k^{2}}, \quad b_{n k}^{-}=\frac{n^{2}-k^{2}}{2}
\end{aligned}
$$

We now turn to the construction of two-point functions between double-trace and single-trace operators. In Appendix A we demonstrate that the two-point function

$$
\left\langle O_{n}^{J}(0): \bar{O}_{m}^{J_{1}} \bar{O}^{J-J_{1}}:(x)\right\rangle \equiv\left\langle O_{n}^{J}(0) \bar{O}_{m s}^{J}(x)\right\rangle
$$

may be obtained from (2.4) by the replacement $x_{12}=x_{13} \rightarrow x, x_{23} \rightarrow \frac{1}{\Lambda}$ so that

$$
\left\langle O_{n}^{J}(0) \bar{O}_{m s}^{J}(x)\right\rangle=g_{2} C_{n m s}\left(1-\lambda^{\prime} \ln (x \Lambda)^{2}\left(a_{n k}+b_{n k}\right)\right) .
$$

The final two-point function that we need involves two double-trace operators. It is straightforward to show that the result up to order $g_{2}$ is

$$
\left\langle: O_{m}^{J_{1}} O^{J-J_{1}}:(0): \bar{O}_{n}^{J_{1}} \bar{O}^{J-J_{1}}:(x)\right\rangle=\delta_{m n}\left(1-\lambda^{\prime} k^{2} \ln \left(x^{2} \Lambda^{2}\right)\right) \text {. }
$$

The complete matrix of two-point functions can now be assembled from (2.2), (2.12) and (2.13). If we introduce the index $A=i, j, m, n$ where $m, n$ index the single-trace operators $O_{n}^{J}$ and $i, j$ index the double-trace operators $O_{m s}^{J}$ so that $i \equiv(m, s)$ then the matrix of two-point functions found in this section can be summarized as

$$
\left\langle O_{A} \bar{O}_{B}\right\rangle=g_{A B}-\lambda^{\prime} h_{A B} \ln x^{2} \Lambda^{2} .
$$

Here $g_{A B}$ gives an inner product on the space of operators (in fact it is just the Hilbert space inner product, according to the state-operator mapping), while $h_{A B}$ is the matrix of anomalous dimensions (obtained by applying the dilatation operator $D=x^{\nu} \partial_{\nu}$ ). See Appendix $\mathrm{C}$ for a schematic discussion of the diagonalization of $\left\langle O_{A} \bar{O}_{B}\right\rangle$. 


\subsection{Construction of eigen-operators}

In order to find eigen-operators we must now diagonalize the matrix of two-point functions. The eigen-operators $\tilde{O}_{n}^{ \pm J}$ and $\tilde{O}_{m s}^{J}$ must, by definition, reduce to $O_{n}^{ \pm J}$ and : $O_{m}^{J_{1}} O^{J-J_{1}}:$ respectively at $g_{2}=0$. These operators take the form

$$
\begin{aligned}
& \tilde{O}_{n}^{ \pm J}=O_{n}^{ \pm J}+g_{2} \sum_{m=0}^{\infty} \sum_{J_{1}=0}^{J} C_{n m s}^{ \pm} M_{n m}: O_{m}^{ \pm J_{1}} O^{ \pm J-J_{1}}:+\cdots \\
& \tilde{O}_{m s}^{ \pm J}=: O_{m}^{ \pm J_{1}} O^{ \pm J-J_{1}}:-g_{2} \sum_{m=0}^{\infty} C_{n m s}^{ \pm} N_{n m} O_{n}^{ \pm J}+\cdots
\end{aligned}
$$

where $M_{n k}$ and $N_{n k}$ are coefficients to be determined. $\tilde{O}_{n}^{ \pm J}$ and $\tilde{O}_{m s}^{ \pm J}$ is easily computed using the results of the previous subsection

$$
\begin{aligned}
\left(4 \pi^{2} x^{2}\right)^{J+2} & \left\langle\tilde{O}_{n}^{ \pm J}(0) \overline{\tilde{O}}_{m s}^{ \pm J}(x)\right\rangle \\
& =g_{2} C_{n m s}^{ \pm}\left[\left(1+M_{n m}-N_{n m}\right)-\lambda^{\prime} \ln (\Lambda x)^{2}\left(a_{n k}^{ \pm}+b_{n k}^{ \pm}+M_{n m} k^{2}-N_{n m} n^{2}\right)\right] .
\end{aligned}
$$

As $\tilde{O}_{n}^{ \pm J}$ and $\tilde{O}_{m s}^{J}$ are eigen-operators in a conformal field theory, 2.16 must vanish identically; this yields the set of simultaneous equations

$$
\begin{array}{r}
1+M_{n m}-N_{n m}=0 \\
a_{n k}^{ \pm}+b_{n k}^{ \pm}+M_{n m} k^{2}-N_{n m} n^{2}=0
\end{array}
$$

which may easily be solved for $M_{n m}$ and $N_{n m}$. Carrying out the algebra we find

$$
\begin{aligned}
& \tilde{O}_{n}^{ \pm J}=O_{n}^{ \pm J}+g_{2} \sum_{m=0}^{\infty} \sum_{J_{1}=0}^{J} C_{n m s}^{ \pm} \frac{a_{n k}^{ \pm}+b_{n k}^{ \pm}-n^{2}}{n^{2}-k^{2}} O_{m}^{ \pm J_{1}} O^{J-J_{1}}+\cdots \\
& \tilde{O}_{m s}^{ \pm J}=: O_{m}^{ \pm J_{1}} O^{J-J_{1}}:-g_{2} \sum_{m=0}^{\infty} C_{n m s}^{ \pm} \frac{a_{n k}^{ \pm}+b_{n k}^{ \pm}-k^{2}}{n^{2}-k^{2}} O_{n}^{ \pm J}+\cdots
\end{aligned}
$$

\subsection{Consistency conditions from three-point functions}

Having determined the BMN eigen-operators to first order in $g_{2} \lambda^{\prime}$, we now turn to the determination of their three-point functions. We will demonstrate that the three-point functions of these operators do indeed take the form required by conformal invariance.

4 The transformation to the \pm basis implies that the summation symbol in (2.15) and other formulas in this basis should be defined as $\sum_{m=0}^{\infty} \equiv \frac{1}{2} \sum_{m=-\infty}^{\infty}$. 
We find that this is true for any value of $b_{n k}$ provided the coefficient $a_{n k}=k^{2}$. This is consistent with (2.6) and provides a check on our algebra.

Combining (2.2), (2.3), (2.4), and (2.18) (and using the fact that double trace correlators factorize to lowest order in $g_{2}$ ) we find

$$
\begin{aligned}
& \left.\left\langle\tilde{O}_{n}^{ \pm J}\left(x_{1}\right) \overline{\tilde{O}}_{m}^{ \pm J_{1}}\left(x_{2}\right) \bar{O}^{J-J_{1}}\right)\left(x_{3}\right)\right\rangle=g_{2} C_{n m s}^{ \pm J} \frac{a_{n k}^{ \pm}+b_{n k}^{ \pm}-k^{2}}{n^{2}-k^{2}} \\
& \times\left(1-\lambda^{\prime}\left[\ln \left|x_{12} \Lambda\right| \frac{2 n^{2} k^{2}-b_{n k}^{ \pm}\left(k^{2}+n^{2}\right)-2 a_{n k}^{ \pm} n^{2}}{k^{2}-a_{n k}^{ \pm}-b_{n k}^{ \pm}}+\ln \left|\frac{x_{13} x_{12} \Lambda}{x_{23}}\right| b_{n k}^{ \pm} \frac{k^{2}-n^{2}}{k^{2}-a_{n k}^{ \pm}-b_{n k}^{ \pm}}\right]\right) .
\end{aligned}
$$

(2.19) takes the standard CFT form for a three-point function between operators of anomalous dimension $n^{2}, k^{2}$, and 0 respectively, provided that

$$
\begin{gathered}
\frac{2 n^{2} k^{2}-b_{n k}^{ \pm}\left(k^{2}+n^{2}\right)-2 a_{n k}^{ \pm} n^{2}}{k^{2}-a_{n k}^{ \pm}-b_{n k}^{ \pm}}=k^{2}+n^{2} \\
b_{n k}^{ \pm} \frac{k^{2}-n^{2}}{k^{2}-a_{n k}^{ \pm}-b_{n k}^{ \pm}}=k^{2} .
\end{gathered}
$$

It is easily verified that the two equations (2.20) are not independent; they are both satisfied for any value of $b_{n k}$ if and only if

$$
a_{n k}^{ \pm}=k^{2}
$$

as promised at the beginning of this subsection.

Notice that the three-point coupling between three normalized eigen-operators involves the coefficient

$$
\tilde{C}_{n m s}^{ \pm}=C_{n m s}^{ \pm} \frac{b_{n k}^{ \pm}}{n^{2}-k^{2}}
$$

which is distinct from the free three-point coupling $C_{n m s}^{ \pm}$even at lowest order in $\lambda^{\prime}$ ! Even though this modification is independent of $\lambda^{\prime}$, it is clearly a quantum effect as the modified three-point function depends on $b_{n k}$, the coefficient of the order $\lambda^{\prime}$ term in (2.7).

\subsection{Anomalous dimensions of BMN eigen-operators}

It is now a simple matter to compute the two-point function of the operator (2.18) and thereby determine its anomalous dimension to order $g_{2}^{2} \lambda^{\prime}$. 5 The two-point functions

5 We do not need to know the form of the operator to order $g_{2}^{2}$ in order to perform this computation. The only order $g_{2}^{2}$ correction to this operator that can contribute to its two-point function at order $g_{2}^{2}$ is a piece proportional to $O_{n}^{J}$; such an addition can be absorbed into a normalization of the operator at this order, and so does not contribute to its scaling dimension. 
of single-trace BMN operators with double-trace operators were presented above while the complete order $\lambda^{\prime} g_{2}^{2}$ two-point functions of single-trace BMN operators were presented in [5, 4] and we reproduce here (with different relative signs than [4]) the relevant equations,

$$
\left\langle O_{n}^{J}(0) \bar{O}_{m}^{J}\right\rangle=\left(\delta_{n m}+g_{2}^{2} A_{n m}\right)\left(1-\left(n^{2}-n m+m^{2}\right) \lambda^{\prime} \ln x^{2} \Lambda^{2}\right)-\frac{g_{2}^{2} \lambda^{\prime}}{4 \pi^{2}} B_{m n} \ln x^{2} \Lambda^{2}
$$

where the matrices $A_{m n}$ and $B_{m n}$ are given by

$$
\begin{gathered}
A_{n m}= \begin{cases}\frac{1}{24} & \text { if } m=n=0 \\
0, & \text { if } m=0, n \neq 0 \text { or } n=0, m \neq 0 \\
\frac{1}{60}-\frac{1}{6 u^{2}}+\frac{7}{u^{4}} & \text { if } m=n \neq 0 \\
\frac{1}{4 u^{2}}\left(\frac{1}{3}+\frac{35}{2 u^{2}}\right) & \text { if } m=-n \neq 0 \\
\frac{1}{(u-v)^{2}}\left(\frac{1}{3}+\frac{4}{v^{2}}+\frac{4}{u^{2}}-\frac{6}{u v}-\frac{2}{(u-v)^{2}}\right) & \text { all other cases }\end{cases} \\
B_{n m}= \begin{cases}0 & \mathrm{n}=0 \text { if } m=0 \\
\frac{1}{3}+\frac{10}{u^{2}} & \text { if } n=m \neq 0 \\
\frac{-15}{2 u^{2}} & \text { if } n=-m \neq 0 \\
\frac{6}{u v}+\frac{2}{(u-v)^{2}} & \text { all other cases }\end{cases}
\end{gathered}
$$

and

$$
u=2 \pi m, \quad v=2 \pi n .
$$

As explained in Appendix C, the anomalous dimension of the $\tilde{O}_{n}^{ \pm J}$ can be readily computed from the diagonal two-point functions $\left\langle\tilde{O}_{n}^{ \pm} \overline{\tilde{O}}_{n}^{ \pm}\right\rangle$. The required algebra is straightforward using the ingredients (2.12), (2.18), (2.22), (2.23), (2.24). The result is

$$
\frac{\Gamma_{n}^{ \pm}}{\lambda^{\prime} g_{2}^{2}}=\mp 2 n^{2} A_{n,-n}+\frac{1}{4 \pi^{2}}\left(B_{n n} \pm B_{n,-n}\right)-\sum_{m=0}^{\infty} \sum_{J_{1}=0}^{J}\left(C_{n m s}^{ \pm}\right)^{2} \frac{\left(b_{n k}^{ \pm}-n^{2}+k^{2}\right)^{2}}{n^{2}-k^{2}}
$$

where we use the value for $b_{n k}^{ \pm}$given in (2.10). Using the identities in Appendix D this may be re-written as

$$
\Gamma_{n}^{ \pm}=g_{2}^{2} \lambda^{\prime} n^{2} A_{n-n}=\frac{g_{2}^{2} \lambda^{\prime}}{4 \pi^{2}}\left(\frac{1}{12}+\frac{35}{32 \pi^{2} n^{2}}\right)
$$

The full anomalous dimension of this operator (including the planar contribution) is

$$
\Delta_{n}^{ \pm}-J-2=\lambda^{\prime} n^{2}\left(1+g_{2}^{2} A_{n,-n}\right)=\lambda^{\prime}\left[n^{2}+\frac{g_{2}^{2}}{4 \pi^{2}}\left(\frac{1}{12}+\frac{35}{32 \pi^{2} n^{2}}\right)\right] .
$$

This is the principal result of this note. 
It is striking that the degeneracy between the even and odd operators (obvious at $\left.g_{2}=0\right)$ persists to order $g_{2}^{2}$. It appears that this degeneracy is a consequence of supersymmetry6; all two-impurity BMN operators lie in a single representation of the ppwave supersymmetry algebra and so are guaranteed to have equal anomalous dimensions. Note that 16 of the 32 supercharges of the $\mathcal{N}=4 d=4$ superconformal algebra commute with $p^{-}=\frac{\mu}{2}(\Delta-J)$; further all supercharges commute with $p^{+}$which is a central element of this algebra in the pp-wave limit. Consequently, states in long representations of the ppwave superalgebra appear in multiplets whose minimum degeneracy is $2^{16 / 2}=16 \times 16$. But this is precisely the number of two-impurity BMN operators; it is natural to conjecture that the two-impurity BMN operators may be related to one another by the action of the 16 supercharges that commute with $p^{-}$; their degeneracy is thus a consequence of supersymmetry. Special examples of such relations have been worked out directly in the gauge theory in [15, [16]. We hope to return to this issue in more detail in future work.

\section{String field theory revisited}

In this section we will use quantum mechanical perturbation theory to compute the energy shifts of a particular set of excited string states in the pp-wave background. This section is almost a direct transcription of Section 5 of [4], now adapted to the $O_{n}^{ \pm J}$ basis.

Utilizing (1.3) and second order non-degenerate perturbation theory (see section 5 of [1]) we find,

$$
\frac{1}{\mu} \sum_{i} \frac{\left|\left\langle n\left|H_{\mathrm{int}}\right| i\right\rangle\right|^{2}}{E_{n}-E_{i}}=g_{2}^{2} \lambda^{\prime} \sum_{i}\left(n^{2}-k^{2}\right) C_{n i}^{2}=-\frac{g_{2}^{2} \lambda^{\prime}}{4 \pi^{2}} B_{n n}=-\frac{g_{2}^{2} \lambda^{\prime}}{4 \pi^{2}}\left(\frac{1}{3}+\frac{10}{v^{2}}\right)
$$

and

$$
\frac{1}{\mu} \sum_{i} \frac{\left\langle n\left|H_{\mathrm{int}}\right| i\right\rangle\left\langle i\left|H_{\mathrm{int}}\right|-n\right\rangle}{E_{n}-E_{i}}=g_{2}^{2} \lambda^{\prime} \sum_{i}\left(n^{2}-k^{2}\right) C_{n i} C_{-n, i}=-\frac{g_{2}^{2} \lambda^{\prime}}{4 \pi^{2}} B_{n,-n}=\frac{g_{2}^{2} \lambda^{\prime}}{4 \pi^{2}} \frac{15}{2 v^{2}}
$$

where we have used $i$ as a collective index for $(m, s)$ as well as for the states in which the $\phi$ and $\psi$ excitations are on different strings, corresponding to the BPS operator : $O_{\phi}^{J_{1}} O_{\psi}^{J-J_{1}}$ :. Unlike in the Yang-Mills calculation of the previous section, the degeneracy between the

6 This explanation was suggested to us by J. Maldacena and M. Van Raamsdonk in response to an earlier version of this paper. 
+ and - sectors of the string theory is thus broken: the correct zeroth-order eigenvectors are

$$
|n, \pm\rangle \equiv \frac{1}{\sqrt{2}}(|n\rangle \pm|-n\rangle)
$$

and their second order energy shifts are

$$
\begin{gathered}
\frac{1}{\mu} \sum_{i} \frac{\left|\left\langle n,+\left|H_{\mathrm{int}}\right| i,+\right\rangle\right|^{2}}{E_{n,+}-E_{i,+}}=-\frac{g_{2}^{2} \lambda^{\prime}}{4 \pi^{2}}\left(B_{n n}+B_{n,-n}\right)=-\frac{g_{2}^{2} \lambda^{\prime}}{4 \pi^{2}}\left(\frac{1}{3}+\frac{5}{2 v^{2}}\right) \\
\frac{1}{\mu} \sum_{i} \frac{\left|\left\langle n,-\left|H_{\mathrm{int}}\right| i,-\right\rangle\right|^{2}}{E_{n,-}-E_{i,-}}=-\frac{g_{2}^{2} \lambda^{\prime}}{4 \pi^{2}}\left(B_{n n}-B_{n,-n}\right)=-\frac{g_{2}^{2} \lambda^{\prime}}{4 \pi^{2}}\left(\frac{1}{3}+\frac{35}{2 v^{2}}\right) .
\end{gathered}
$$

Sadly, neither of these energy shifts agrees with the anomalous dimension computed in the previous section.

It is possible that this disagreement is resolved by the presence of an explicit quadratic contact term in the string field theory Hamiltonian. In order to restore the degeneracy of string states and resolve the discrepancy, this contact term would have to have matrix elements

$$
\begin{gathered}
\frac{1}{\mu}\left\langle n,+\left|V_{2}\right| n,+\right\rangle=\frac{g_{2}^{2} \lambda^{\prime}}{4 \pi^{2}}\left(\frac{5}{12}+\frac{55}{8 v^{2}}\right) \\
\frac{1}{\mu}\left\langle n,-\left|V_{2}\right| n,-\right\rangle=\frac{g_{2}^{2} \lambda^{\prime}}{4 \pi^{2}}\left(\frac{5}{12}+\frac{175}{8 v^{2}}\right) .
\end{gathered}
$$

Since these expressions are positive it is at least possible that they come from a contact term in the string field theory Hamiltonian, which would be determined by the anti-commutator of two supersymmetry generators [17, 18, 19]. It would be very interesting to compare (3.5) with a direct computation of contact terms in string field theory.

It should also be mentioned that the energy shifts could possibly receive contributions at this order from states with 4 or more impurities, due to enhanced matrix elements between states with different numbers of impurities, as reported in [7]. Note, however, that this effect could not alone cure the apparent discrepancy between string theory and gauge theory, since the contributions, if any, due to such massive intermediate states would be negative.

\section{Acknowledgements}

We would like to thank V. Khoze, C. Kristjansen, L. Motl, J. Plefka, A. Postnikov, G. Semenoff, W. Skiba, M. Spradlin, M. Staudacher, A. Volovich and especially J. Maldacena, M. Van Raamsdonk and H. Verlinde for useful discussions and correspondence. S. M. would 
like to thank the Tata Institute for Fundamental Research, Mumbai for hospitality while this work was being completed. S.M. and D.F.Z would like to thank the organizers of the Amsterdam Summer workshop for hospitality while this work was in progress. N.R.C. and D.Z.F. are supported by the NSF under PHY 00-96515 and the DOE under DF-FC0294ER40818. N.R.C. is also supported by NSERC of Canada. M.H. is supported in part by the DOE under grant DE-FG01-91ER40654 and in part by the Packard Foundation. S.M. is supported by a Harvard Junior Fellowship and by the DOE grant DE-FG01-91ER40654.

\section{Appendix A. The space-time structure of two- and three-point functions}

In this appendix we will justify the space time dependence of the order $\lambda^{\prime}$ terms in the equation (2.4) for the three-point function $\left\langle O_{n}^{J}\left(x_{1}\right) \bar{O}_{m_{1}}^{J_{1}}\left(x_{2}\right) \bar{O}^{J_{2}}\left(x_{3}\right)\right\rangle$, and discuss its relation to mixed two-point functions as in (2.12). There are two distinct structures coming from the quartic interactions in contributing Feynman diagrams. The first structure occurs

in diagrams in which one pair of lines from the operator $O_{n}^{J}\left(x_{1}\right)$ and one pair from $\bar{O}_{m_{1}}^{J_{1}}\left(x_{2}\right)$ terminate at the interaction vertex at $z$. This leads to the the same space time integral which occurs in two-point functions, namely

$$
\int \frac{d^{4} z}{z^{4}\left(z-x_{12}\right)^{4}}=2 \pi^{2} \frac{\ln \left(x_{12}^{2} \Lambda^{2}\right)}{x_{12}^{4}} .
$$

The second structure occurs in diagrams where lines from all three operators are connected to the interaction vertex. This leads to the integral

$$
\int \frac{d^{4} z}{z^{4}\left(x_{12}-z\right)^{2}\left(x_{13}-z\right)^{2}}=\pi^{2} \ln \left(\frac{x_{12}^{2} x_{13}^{2} \Lambda^{2}}{x_{23}^{2}}\right)
$$

This integral was evaluated using dimensional regularization in [20] and with differential regularization [21] in our work. The integrals above determine the general structure in (2.4), and the specific values of the parameters $a_{n k}, b_{n k}$ which come from our summation of the contributing diagrams are given in (2.6).

The two-point function $\left\langle O_{n}^{J}\left(x_{1}\right): \bar{O}_{m_{1}}^{J_{1}} \bar{O}^{J_{2}}:\left(x_{2}\right)\right\rangle$ can be obtained from the threepoint function in the OPE limit $x_{3} \rightarrow x_{2}$. See [22]. It can also be found simply by setting $x_{13}=x_{12}$ and $x_{23}=1 / \Lambda$, since (A.2) reduces to (A.1) if this is done. Two-point functions were also obtained directly from summation of Feynman diagrams in our work. 


\section{Appendix B. Absence of mixing with BPS double-trace operators}

In [4] - see also [23,20] - non-vanishing three-point functions $\left\langle O_{n}^{J} \bar{O}_{\phi}^{J_{1}} \bar{O}_{\psi}^{J-J_{1}}\right\rangle$ were computed, where $O_{\phi}^{J}=\operatorname{Tr}\left(\phi Z^{J}\right) / \sqrt{N^{J+1}}$ and $O_{\psi}^{J}=\operatorname{tr}\left(\psi Z^{J}\right) / \sqrt{N^{J+1}}$ are BPS operators. The corresponding dual two-string state contributed to the sum over intermediate states in the consistency check in [4]. One may then wonder why the double-trace : $\bar{O}_{\phi}^{J_{1}} \bar{O}_{\psi}^{J-J_{1}}$ : was not included in the initial ansatz (2.18) for our mixing calculation, and we now justify its omission.

Let $O^{J}=\operatorname{Tr}\left(Z^{J}\right) / \sqrt{J N^{J}}$ denote the chiral primary operator. It is quite easy to verify that the linear combination

$$
O_{\text {susy }}^{J} \equiv\left(\sqrt{\frac{J_{1}-1}{J_{2}+1}}: O_{0}^{J_{1}-1} O^{J_{2}+1}:+: O_{\phi}^{J_{1}} O_{\psi}^{J_{2}}:\right)+\left(J_{1} \leftrightarrow J_{2}\right)
$$

is an $S U(4)$ descendant of the chiral primary : $\operatorname{Tr}\left(Z^{J_{1}+1}\right) \operatorname{Tr}\left(Z^{J_{2}+1}\right):$. Thus the operator $O_{\text {susy }}^{J}$ decouples from $O_{n}^{J}$ and from : $O_{m}^{J_{1}} O^{J_{2}}$ : for $n, m \neq 0$. The decoupling can easily be observed; the appropriate combination of mixed two-point functions which can be obtained from the three-point functions given in (3.10) and (3.11) of [4 vanish. 7 The decoupling means that we can simply omit the operators : $\bar{O}_{\phi}^{J_{1}} \bar{O}_{\psi}^{J_{2}}$ : from the mixing calculation, if we keep : $O_{0}^{J_{1}} O^{J_{2}}$ :. However, one may also observe directly from the solution for $\tilde{O}_{n}^{J}$ in (2.18) that : $O_{0}^{J_{1}} O^{J_{2}}$ : also decouples.

\section{Appendix C. Diagonalization procedure}

In this appendix we provide an overview of the diagonalization procedure used to obtain the eigen-operators in (2.18) and their scale dimension.

The matrix of two point functions is given in (2.14) The problem is to diagonalize the matrix of anomalous scaling dimensions $h_{A B}$ relative to the inner product $g_{A B}$. Both $h_{A B}$ and $g_{A B}$ are functions of $g_{2}$ and we will perform our analysis up to and including contributions at order $g_{2}^{2}$. To explain the notion of relative diagonalization, we note that the goal is to find a set of operators,

$$
\tilde{O}_{A}=O_{B} S_{A}^{B}
$$

7 To correct an error the right side of (3.11) in $₫$ should be multiplied by -1 . 
and numbers $\gamma_{A}$ such that

$$
\left(4 \pi^{2} x^{2}\right)^{J+2}\left\langle\tilde{O}_{A}(0) \overline{\tilde{O}}_{B}(x)\right\rangle=\delta_{A B}\left(1-\lambda \gamma_{A} \ln x^{2} \Lambda^{2}\right)
$$

Hence the matrix $S$ must satisfy

$$
S^{\dagger} g S=I, \quad S^{\dagger} h S=\gamma
$$

where $\gamma_{A B}=\gamma_{A} \delta_{A B}$ is the diagonalized matrix of anomalous scaling dimensions. Together these imply

$$
S^{-1} g^{-1} h S=\gamma
$$

Thus we must find the eigenvalues and eigenvectors of the matrix

$$
h^{A}{ }_{C}=g^{A B} h_{B C},
$$

where $g^{A B}$ is the inverse matrix of $g_{A B}$. This diagonalization process is essentially equivalent to non-degenerate quantum mechanical perturbation theory. We use a two-step process in which we first find the eigenvectors to order $g_{2}$, and then use the two-point function of the eigen-operators to compute the scale dimension to order $g_{2}^{2}$.

To make the method clear consider the toy eigen-value problem

$$
M^{a}{ }_{b} V_{(c)}^{b}=\lambda_{(c)} V_{(c)}^{a} .
$$

Diagonal elements are given by $M^{a}{ }_{a}=\rho_{(a)}$, and we wish to work to first order in all off-diagonal elements. To zero order, the eigenvalues are $\lambda_{(c)}=\rho_{(c)}$ and eigenvectors are $V_{(c)}^{a}=\delta^{a}{ }_{c}$. It is then trivial to see that the first order eigenvectors are

$$
\begin{aligned}
& V_{(c)}^{a}=1 \text { if } a=c \\
& V_{(c)}^{a}=\frac{M^{a}{ }_{c}}{\rho_{(c)}-\rho_{(a)}} \text { if } a \neq c
\end{aligned}
$$

The correction is well-defined if there is no degeneracy. This toy model is pertinent to our block matrix $h^{A}{ }_{B}$ because the non-diagonal corrections to $h^{n}{ }_{m}$ and $h^{i}{ }_{j}$ are of order $g_{2}^{2}$ and can be temporarily ignored. One immediately finds the (dominantly single-trace) eigenvectors to first order in $g_{2}$ :

$$
\tilde{O}_{n}=O_{n}+\sum_{i} \frac{O_{i} h_{n}^{i}}{n^{2}-k^{2}}=O_{n}-g_{2} \sum_{i} \frac{k}{n+k} C_{n, i} O_{i} .
$$


One may also write an analogous expression for the dominantly double-trace eigenvectors of the structure $\tilde{O}_{i}=O_{i}+\mathcal{O}\left(g_{2} O_{m}\right)$. From linear combinations of $O_{n}$ and $O_{-n}$ one can easily obtain the eigen-operators in (2.18) (with (2.6) inserted).

The second step of our procedure is based on the matrix of two-point functions $\left\langle\tilde{O}_{A} \overline{\tilde{O}}_{B}\right\rangle$ (in the \pm basis). Because of the approximate diagonalization, off-diagonal entries $\left\langle\tilde{O}_{n} \overline{\tilde{O}}_{i}\right\rangle$ are of order $g_{2}^{3}$ and can be dropped. We can confine our attention to the upper block of the matrix which is again of the form

$$
\left\langle\tilde{O}_{n}^{ \pm} \tilde{O}_{m}^{ \pm}\right\rangle=G_{n m}-\lambda H_{n m} \ln x^{2}
$$

(with superscripts omitted on the RHS to avoid clutter). We need to think about diagonalizing the matrix $H_{m}^{n}=G^{n p} H_{p m}$, in which $G_{n m}, H_{n m}$ have the structure

$$
\begin{aligned}
& G_{n m}=\delta_{n m}+g_{2}^{2} g_{m n}^{\prime} \\
& H_{n m}=n^{2} \delta_{n m}+g_{2}^{2} h_{n m}^{\prime}
\end{aligned}
$$

The off-diagonal elements of $H_{m}^{n}$ are of order $g_{2}^{2}$. There is no degeneracy so these affect eigenvalues beginning only in order $g_{2}^{4}$. Much as in the toy model above, the anomalous dimension of the eigen-operator $\tilde{O}_{n}^{ \pm}$is then simply the diagonal element

$$
H_{n}^{n}=n^{2}+g_{2}^{2}\left(h_{n n}^{\prime}-n^{2} g_{n n}^{\prime}\right) .
$$

It is this quantity in the \pm channels that is computed in (2.25) .

\section{Appendix D. Deconstruction identities}

In this appendix we note several useful identities which relate the three point and twopoint coefficients that appear in this paper. We follow the notation of section 3 in which $i$ is used as a collective index labeling both types of double-trace operators: : $O_{m}^{J_{1}} O^{J-J_{1}}$ : (for which $s=J_{1} / J$ and $k=m / s$ ) and $: O_{\phi}^{J_{1}} O_{\psi}^{J-J_{1}}:$ (for which $s=J_{1} / J$ and $k=0$ ). We then have:

$$
\begin{aligned}
\sum_{i} C_{n i} C_{m i} & =2 A_{n m} \\
\sum_{i} k C_{n i} C_{m i} & =(n+m) A_{n m} \\
\sum_{i} k^{2} C_{n i} C_{m i} & =\left(n^{2}+m^{2}\right) A_{n m}+\frac{1}{4 \pi^{2}} B_{n m} .
\end{aligned}
$$


The first of these was pointed out in [8]. The first and last can be combined to obtain the relation

$$
\sum_{i}\left(n^{2}-k^{2}\right) C_{n i}^{2}=-\frac{1}{4 \pi^{2}} B_{n n}
$$

which is the content (up to a sign) of the "unitarity check" of [4].

These formulas are not numerical coincidences, nor do they in and of themselves provide evidence for the BMN correspondence, since they can be derived purely within the gauge theory. Nonetheless the derivation, which we will explain in this appendix, naturally calls to mind a picture of interacting strings, and may in the future be useful in gaining a deeper understanding of the correspondence.

We imagine the string of $Z \mathrm{~s}$ in the operator $O_{n}^{J}$ to be a circle on which the $\phi$ and $\psi$ impurities are quantum mechanical particles moving with momentum $n$ and $-n$ respectively - their wave function is the weight of each possible configuration in the definition of the operator (1.2). (Morally this point of view is a "de-second quantization" of the worldsheet field theory, which is appropriate in the large $\mu$ limit where the excitations are very massive and we are fixing their number and type.) A free torus diagram contributing to $A_{n m}$ can be represented by a unitary operator $T\left(s, j_{1}, j_{3}\right)$ which acts on this 2-particle Hilbert space by dividing the circle (of length 1, for simplicity) into 4 blocks of lengths $j_{1}, j_{2}, j_{3}, j_{4}$ (where $j_{2}=s-j_{1}, j_{4}=1-s-j_{3}$ ) and transposing blocks 1 and 2 and blocks 3 and 4 (see figure 3 of 四, for example). We thus have

$$
A_{n m}={ }_{\phi}\left\langle\left. n\right|_{\psi}\langle-n|R| n\rangle_{\phi} \mid-n\right\rangle_{\psi}
$$

where

$$
R=\frac{1}{4} \int_{0}^{1} d s \int_{0}^{s} d j_{1} \int_{0}^{1-s} d j_{3} T\left(s, j_{1}, j_{3}\right)
$$

(the integrals count each diagram 4 times, hence the 1/4). But if we imagine pinching the circle into two circles of lengths $s$ and $1-s, T\left(s, j_{1}, j_{3}\right)$ acts by rotating the $s$ circle through an angle $2 \pi j_{1}$ and the $1-s$ circle through an angle $2 \pi j_{3}$. The integration over these rotation angles will ensure a factorization over physical intermediate states. Let us see this more explicitly.

In such a pinching, the 2-particle Hilbert space decomposes into a direct sum of four Hilbert spaces depending on which little circle each impurity is on:

$$
\mathcal{H}_{\phi}(1) \mathcal{H}_{\psi}(1)=\mathcal{H}_{\phi}(s) \mathcal{H}_{\psi}(s) \oplus \mathcal{H}_{\phi}(1-s) \mathcal{H}_{\psi}(1-s) \oplus \mathcal{H}_{\phi}(s) \mathcal{H}_{\psi}(1-s) \oplus \mathcal{H}_{\phi}(1-s) \mathcal{H}_{\phi}(s)
$$


The integral over the rotation angle $j_{1}$ projects out any state with non-zero total momentum on the $s$ circle, and similarly the integral over $j_{3}$ projects out any state with non-zero total momentum on the $1-s$ circle. Hence we have

$$
\int_{0}^{s} d j_{1} \int_{0}^{1-s} d j_{3} T\left(s, j_{1}, j_{3}\right)=s(1-s)\left(\sum_{m} P_{m}(s)+\sum_{m} P_{m}(1-s)+P_{0^{\prime}}(s)+P_{0^{\prime}}(1-s)\right),
$$

where $P_{m}(s)$ projects onto the state $|m, s\rangle_{\phi}|-m, s\rangle_{\psi}$ corresponding to the operator : $O_{m}^{J_{1}} O^{J-J_{1}}$ :, and $P_{0^{\prime}}(s)$ projects onto the state $|0, s\rangle_{\phi}|0,1-s\rangle_{\psi}$ corresponding to the operator : $O_{\phi}^{J_{1}} O_{\psi}^{J-J_{1}}:$. Using

$$
C_{n i}=\sqrt{s(1-s)} \begin{cases}{ }_{\phi}\left\langle\left. n\right|_{\psi}\langle-n \mid m, s\rangle_{\phi} \mid-m, s\right\rangle_{\psi} & \text { for : } O_{m}^{J_{1}} O^{J-J_{1}}: \\ { }_{\phi}\left\langle\left. n\right|_{\psi}\langle-n \mid 0, s\rangle_{\phi} \mid 0,1-s\right\rangle_{\psi} & \text { for : } O_{\phi}^{J_{1}} O_{\psi}^{J-J_{1}}:\end{cases}
$$

we recover the first identity of (D.1).

Inserting $k^{2} / 2$ in the sum means including interaction vertices in the diagrams. However, the vertex is included only if it is between two lines on the same small circle. Thus (in the language of [4]) all the nearest neighbor interactions are counted, but only half the semi-nearest neighbor interactions. The non-nearest neighbor interactions are also under counted by a factor of 2 , but this is a little more complicated: only $1 / 3$ of them are counted, but overall diagrams with three blocks are over counted by a factor of $3 / 2$. Hence we have

$$
\frac{1}{2} \sum_{i} k^{2} C_{n i} C_{m i}=n m A_{n m}+\frac{1}{2}(n-m)^{2} A_{n m}+\frac{1}{8 \pi^{2}} B_{n m}
$$

which is equivalent to the last identity in (D.1).

The second identity of (D.1) can also be derived by similar but more involved reasoning.

\section{Appendix E. Degenerate operators and mixing}

The diagonalization process is an application of non-degenerate quantum mechanical perturbation theory. A potential complication occurs because single and double-trace operators have equal zeroth-order eigenvalues when $n=k$, threatening a divergence in the calculation of eigenvectors to order $g_{2}$. This would signal the breakdown of non-degenerate perturbation theory and the necessity of using the degenerate theory. As one can see from the right-hand side of (2.18), such a divergence does not occur, thanks to some rather 
delicate cancellations. These cancellations have an interesting reflection in the dual string theory [4. The amplitude for an excited string state corresponding to a 2 impurity BMN operator to decay into two particles vanishes onshell at lowest order in $\frac{1}{\mu}$; thus these excited string states are stable to lowest order in $g_{2}$.

Let us now consider higher rank $r \geq 3$ multi-trace operators which we call $O_{r}$ for short. The two-point functions $\left\langle\bar{O}_{n} O_{r}\right\rangle$ are of order $g_{2}^{r-1}$. Generically such operators contribute to eigenvalues beginning in higher order than $g_{2}^{2}$. There could be a complication for triple-trace operators $O_{3}=: O_{m}^{J_{1}} \operatorname{Tr}\left(Z^{J_{2}}\right) \operatorname{Tr}\left(Z^{J_{3}}\right)$ : which are degenerate with singletraces when $n=m / s$. If the relevant interaction matrix elements do not vanish, eigenoperators change at zeroth order and scale dimensions at order $g_{2}^{2}$. The vanishing or otherwise of these interaction matrix elements is linked to the vanishing or otherwise of the onshell one-three particle decay amplitudes of string field theory.

Degeneracy cannot occur for momentum $n=1$ since $m \in Z^{+}$and $0<J_{1}<J$. A calculation of two-point functions $\left\langle\bar{O}_{n} O_{3}\right\rangle$ to order $g_{2}^{2}$ and $\lambda^{\prime} g_{2}^{2}$ would be needed to see if the interaction matrix elements vanish, as they do for double-traces. The calculation has not been done yet, so a conservative position is that present mixing results are valid for momentum $n=1$ where degeneracy cannot occur, but may need modification for $n \geq 2$ if such mixing does occur. 


\section{References}

[1] D. Berenstein, J. M. Maldacena and H. Nastase, "Strings in flat space and pp waves from N = 4 super Yang Mills," JHEP 0204, 013 (2002) arXiv:hep-th/0202021.

[2] C. M. Hull, "Killing Spinors And Exact Plane Wave Solutions Of Extended Supergravity," Phys. Rev. D 30, 334 (1984). M. Blau, J. Figueroa-O'Farrill, C. Hull and G. Papadopoulos, "A new maximally supersymmetric background of IIB superstring theory," JHEP 0201, 047 (2002) arXiv:hep-th/0110242]. ;M. Blau, J. Figueroa-O'Farrill, C. Hull and G. Papadopoulos, "Penrose limits and maximal supersymmetry," Class. Quant. Grav. 19, L87 (2002) arXiv:hep-th/0201081.

[3] R. R. Metsaev, "Type IIB Green-Schwarz superstring in plane wave Ramond-Ramond background," Nucl. Phys. B 625, 70 (2002) arXiv:hep-th/0112044. R. R. Metsaev and A. A. Tseytlin, "Exactly solvable model of superstring in plane wave RamondRamond background," Phys. Rev. D 65, 126004 (2002) arXiv:hep-th/0202109.

[4] N. R. Constable, D. Z. Freedman, M. Headrick, S. Minwalla, L. Motl, A. Postnikov and W. Skiba, "PP-wave string interactions from perturbative Yang-Mills theory," JHEP 0207, 017 (2002) arXiv:hep-th/0205089.

[5] C. Kristjansen, J. Plefka, G. W. Semenoff and M. Staudacher, "A new double-scaling limit of N $=4$ super Yang-Mills theory and PP-wave strings," arXiv:hep-th/0205033.

[6] D. Berenstein and H. Nastase, "On lightcone string field theory from super Yang-Mills and holography," arXiv:hep-th/0205048.

[7] M. Spradlin and A. Volovich, "Superstring interactions in a pp-wave background II," arXiv:hep-th/0206073.

[8] M. x. Huang, "String interactions in pp-wave from $\mathrm{N}=4$ super Yang Mills," arXiv:hepth/0206248.

[9] P. Lee, S. Moriyama and J. w. Park, "Cubic interactions in pp-wave light cone string field theory," arXiv:hep-th/0206065.

[10] Y. j. Kiem, Y. b. Kim, S. m. Lee and J. m. Park, "pp-wave / Yang-Mills correspondence: An explicit check," arXiv:hep-th/0205279.

[11] M. Spradlin and A. Volovich, "Superstring interactions in a pp-wave background," arXiv:hep-th/0204146.

[12] M. Bianchi, B. Eden, G. Rossi and Y. S. Stanev, "On operator mixing in N = 4 SYM," arXiv:hep-th/0205321.

[13] N. Beisert, C. Kristjansen, J. Plefka, G. W. Semenoff and M. Staudacher, "BMN Correlators and Operator Mixing in N=4 Super Yang-Mills Theory," arXiv:hepth/0208178.

[14] D. J. Gross, A. Mikhailov and R. Roiban. "A calculation of the plane wave string Hamiltonian from N=4 super-Yang-Milss theory," archiv:hep-th/0208231

[15] A. Santambrogio and D. Zanon, arXiv:hep-th/0206079. 
[16] U. Gürsoy, "Vector operators in the BMN correspondence," arXiv:hep-th/0208041.

[17] J. Greensite and F. R. Klinkhamer, "Superstring Amplitudes And Contact Interactions," Nucl. Phys. B 304, 108 (1988).

[18] M. B. Green and J. H. Schwarz, "Superstring Interactions," Nucl. Phys. B 218, 43 (1983).

[19] H. Verlinde, "Bits, matrices and 1/N," arXiv:hep-th/0206059.

[20] C. S. Chu, V. V. Khoze and G. Travaglini, "Three-point functions in N = 4 Yang-Mills theory and pp-waves," JHEP 0206, 011 (2002) arXiv:hep-th/0206005.

[21] D. Z. Freedman, K. Johnson and J. I. Latorre, "Differential regularization and renormalization: A New method of calculation in quantum field theory," Nucl. Phys. B 371, 353 (1992).

[22] C. S. Chu, V. V. Khoze and G. Travaglini, "pp-wave string interactions from n-point correlators of BMN operators," arXiv:hep-th/0206167.

[23] M. x. Huang, "Three point functions of $\mathrm{N}=4$ super Yang Mills from light cone string field theory in pp-wave," Phys. Lett. B 542, 255 (2002) arXiv:hep-th/0205311. 